related to physical problems, self-esteem, emotional effects on parents and family activities. A psychosocial summary score was significantly lower for children with ADHD, compared with asthmatic children. (Escobar R, Soutullo CA, Hervas A et al. Worse quality of life for children with newly diagnosed attention-deficit/hyperactivity disorder, compared with asthmatic and healthy children. Pediatrics September 2005;116:e364-e369). (Respond: Rodrigo Escobar MD, Avenida de la Industria, 30, 28108 Alcobendas (Madrid), Spain. Email: escobar_rodrigo@lilly.com).

COMMENT. Children with untreated ADHD have problems with quality of life that affect their behavior, self-esteem, psychosocial and physical functioning, and their relation to parents and families. The findings demonstrate the importance of early recognition and treatment of ADHD.

\title{
MOVEMENT AND MOTOR DISORDERS
}

\section{BOBBLE-HEAD DOLL SYNDROME TREATED SURGICALLY}

A case of a 4-year-old child with bobble-head doll syndrome (BHDS) caused by a suprasellar arachnoid cyst and successfully treated by endoscopic cystovenriculostomy is reported from Medisch Centrum Haaglanden, The Hague; and Academical Hospital Nijmegen, The Netherlands. The cyst collapsed and the aqueductal obstruction resolved. Early surgical treatment of BHDS with suprasellar cyst is recommended to prevent permanent neurologic damage and psychomotor retardation. (Hagebeuk EEO, Kloet A, Grotenhuis JA, Peeters EAJ. Bobble-head doll syndrome successfully treated with an endoscopic ventriculocystocisternostomy. J Neurosurg (Pediatrics 3) September 2005;103:253-259). (Eveline EO Hagebeuk MD, Dept Child Neurology, Emma Children Hospital/AMC (G8-205), PO Box 22660, 1100 DD Amsterdam, the Netherlands).

COMMENT. Endoscopic treatment of a suprasellar arachnoid cyst relieved aquductal obstruction, and an associated bobble-head doll syndrome resolved. BHDS, characterized by a $2-3 \mathrm{~Hz}$ back-and-forth head movement, is caused by a suprasellar arachnoid or third ventricular cyst, and less often is secondary to aqueductal stenosis or VP shunt dysfunction. The tremor disappaars in sleep and is increased by walking and excitement. It affects children younger than 10 years of age, and may present with macrocephaly, symptoms of raised intracranial pressure, optic nerve atrophy, ataxia, endocrine disorders (small stature, obesity, precocious puberty) or mental retardation. Thirty cases have been reported since the first description by Benton JW and associates (Neurology 1966;16:725-729), and are reviewed by the authors.

\section{CEREBRAL PALSY DEFINITION AND CLASSIFICATION}

A revised definition and classification of cerebral palsy is proposed by the Executive Committee of an International Workshop held in Betheda, MD, 2004. (Goldstein M et al. Dev Med Child Neurol August 2005;47:571-576; and 508-510). (E-mail: mgoldstein@ucp.org) 